\title{
Multi-feature Fusion for PoISAR Image Classification of Oil Slick Thickness
}

\author{
Shiyi Hong ${ }^{1, a^{*}, ~ H a o ~ G u o}{ }^{2, b}$ and Jubai $\mathrm{An}^{3, \mathrm{c}}$ \\ ${ }^{1}$ Dalian Maritime University, Dalian 116026, China \\ 2 Dalian Maritime University, Dalian 116026, China \\ ${ }^{3}$ Dalian Maritime University, Dalian 116026, China \\ a714986236@qq.com, bguohao0512@hotmail.com, cjubaian@dlmu.edu.cn
}

Keywords: Polarimetric Synthetic Aperture Radar (PoISAR); Multi-feature fusion; Oil slick; Image classification

\begin{abstract}
The oil slick outline and the information of thickness are important indicators of estimating oil spill. How to estimate the oil slick thickness quickly is a hot research topic. In this paper, multi-feature fusion strategy is used to design classifier based on the potential correlation between Polarimetric Synthetic Aperture Radar (PolSAR) characteristics and oil slick thickness. Taking into account the correlation between polarization characteristics, Mahalanobis distance is used to optimize initial cluster center of fuzzy $\mathrm{C}$-means clustering and then, the estimation of oil slick thickness is carried out. The algorithm is proved to be effective by classifying the oil slick thickness of two groups of PolSAR oil spill data in Mexico Bay.
\end{abstract}

\section{Introduction}

Affected by factors such as wind or currents, oil spill on the sea surface has a strong timeliness [1]. It's difficult to assure the synchronism and quasi synchronism of data, which makes the model applicability in doubt [2]. Reza Shirvany etc. studied that the degree of depolarization reveals the oil structures and properties in different dual-pol modes with $(\mathrm{HH}, \mathrm{VV})$ giving the maximum depolarization contrast between dark oil (Thicker oil layers) and clear water in 2012 [3]. It shows that there is correlation between characteristics of PolSAR and the oil slick thickness.

This paper adopts multi-feature fusion strategy to design the classifier based on the potential correlation between PolSAR characteristics and oil slick thickness. Mahalanobis distance is used to optimize the initial cluster center of fuzzy $\mathrm{C}$-means clustering and then, the estimation of oil slick thickness is carried out. The algorithm is proved to be effective by classifying the oil slick thickness of two groups of PolSAR oil spill data in Mexico Bay.

\section{Image Data Sets}

This study uses two sets of data: (1) quasi synchronous dual polarization SAR (HH-HV) and MODIS data for the selection of polarization characteristics; (2) two scenes of PolSAR oil spill data tested for verifying the correlation between the characteristics and the oil film thickness.

Quasi synchronous dual polarization SAR (HH-HV) and MODIS data. This study uses the quasi synchronous dual polarization SAR and MODIS data of the Mexico Bay oil spill (as shown in Fig. 1) to analyze and select the polarization characteristics based on the correlation between the polarization characteristics and the oil slick thickness. For the MODIS multi-spectral data, in the early stage of the study, the oil slick thickness was classified by the first, 7th, 10th, 18th, 26th band on the basis of removing the distortion band, and the oil slick thickness was divided into 4 stages.

Two Sets of PolSAR oil spill data from Mexico Bay. In order to verify the correlation between the characteristics and the oil slick thickness and the estimating effect of oil slick thickness by the feature fusion, two scenes of PolSAR oil spill data, obtained in Mexico bay in May, 2010, are adopted to conduct experiments (as shown in Fig. 2). The Mexico Bay data (No.1), obtained on May 8th, 2010, includes a larger area of oil spill and a spreading area of the slick. The Mexico Bay data (No.2), obtained 
on August 24th, 2011, shows that oil slick is relatively thin as a whole and the area of oil presents a dispersed state.

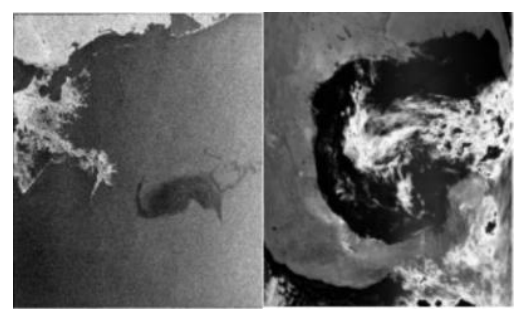

(a)

(b)

Figure 1. The oil spill data of Mexico Bay. (a)SAR image (2010-04-28 T11:51:59), (b)

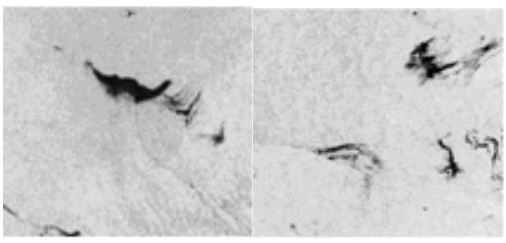

(a)

(b)

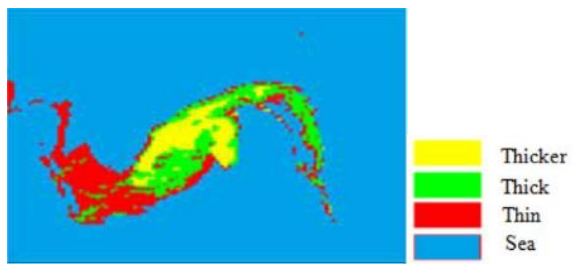
Mexico Bay. (a)No.1, (b) No.2

Figure 3. Thickness classification of oil spill in Mexico Bay

MODIS image (the 7th band)

(2010-04-28 T13:21:23)

\section{SAR Polarization Characteristics Analysis and Selection}

Sampling. The classification result of oil slick thickness in MODIS data is shown in Fig. 3 in Mexico Bay on April 28th, 2010. There are 3 different types in the study. Training samples and test samples are from a series of channels which include the selected features. The sampling positions include forty-five sub-images (fifteen sub-images for each type). Training samples are selected in specific sampling positions by dividing the sub-image into sixteen smaller patches. In this way there are 240 smaller patches for each type of every single feature (120 as training samples and 120 as test samples).

Feature Selection. Polarimetric features and polarimetric interferometric features which are related to the structure and complexity of the observed objects are from polarimetric decomposition. There are many different types of polarimetric decomposition, which can be divided into two groups: (1) coherent target decomposition with target scattering matrix, and (2) non-coherent target decomposition based on polarimetric target covariance matrix, polarimetric coherent matrix, Mueller matrix or Stokes matrix. Those complex scattering behavior of the target can be viewed as a random process, and the premise of coherent or pure scatters cannot be guaranteed, therefore, the polarimetric features in current study are selected from the non-coherent target decomposition. Based on quasi synchronous dual polarization SAR (HH-HV) and MODIS data, feature selection is performed in the study. The backscattering coefficients of $\mathrm{HH}$ and $\mathrm{HV}, \sigma^{H H}$ and $\sigma^{H V}$, can be used as two candidate features. Duan etc. used cross-polarization ration of SAR for discriminating between mineral oil and biogenic oil, so the study uses cross-polarization ration as a candidate feature. Here are a lot of studies on the Cloude decomposition ( $H / \alpha$ ) method of dual-polarizain SAR. Wang, et al. provided a $H / \alpha$ decomposition method of ALOS SAR (HH-HV) for the vegetation classification. The scattering entropy is introduced to analyze the disorder of terrestrial object scattering process, and the scattering angle $\alpha$ represents the average scattering mechanism from surface scattering to dihedral scattering.

Simple Discrimination Analysis. To assess discrimination ability of those features, a simple discrimination analysis based on Bhattacharyya distance. The simple discrimination analysis is created by calculating the distance between a test sample and all training samples. Classification of the test samples produces confusion matrix. A preferred features subset may have the two advantages:

- The Kappa coefficients $(\kappa)$ of a feature is greater than 0.4 , which indicates moderate or higher discrimination capability.

- The complementary relationship exists among different features.

There are four features $\left(\sigma^{H H}, d, H\right.$ and $\alpha$ ) whose Kappa coefficient is higher than 0.4 in Fig. 4.

Summary. Based on the quasi synchronous dual polarization SAR and multi-spectral MODIS oil spill data, a feature analysis and selection method by Bhattacharyya distance is designed in the chapter. 
Some polarization characteristics of the higher correlation degree with oil slick thickness are obtained based on this method.

Table 1 High and low settings of predictor variables

\begin{tabular}{|l|l|l|l|l|l|l|}
\hline Type & $\begin{array}{c}\text { The } \\
\text { thickes } \\
\text { t }\end{array}$ & $\begin{array}{c}\text { The } \\
\text { thicker }\end{array}$ & $\begin{array}{c}\text { The } \\
\text { thinner }\end{array}$ & $\begin{array}{c}\text { The } \\
\text { thinnest }\end{array}$ & Sum & $\begin{array}{c}\text { Precis } \\
\text { ion }\end{array}$ \\
\hline $\begin{array}{l}\text { The } \\
\text { thickest }\end{array}$ & 3525 & 100 & 206 & 111 & 3942 & 89.42 \\
\hline $\begin{array}{l}\text { The } \\
\text { thicker }\end{array}$ & 176 & 4631 & 291 & 281 & 5379 & 86.09 \\
\hline $\begin{array}{l}\text { The } \\
\text { thinner }\end{array}$ & 185 & 177 & 4558 & 165 & 5085 & 89.64 \\
\hline $\begin{array}{l}\text { The } \\
\text { thinnest }\end{array}$ & 129 & 151 & 275 & 4639 & 5194 & 89.31 \\
\hline
\end{tabular}

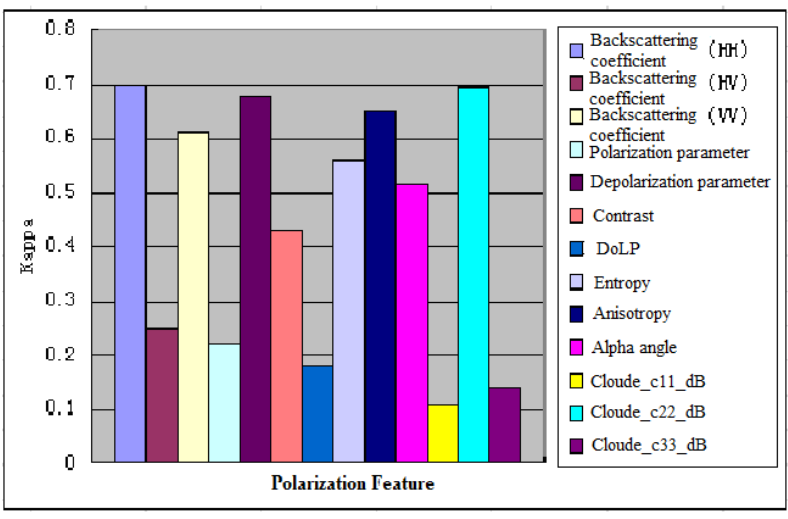

Figure 4. Thickness classification of oil spill in Mexico Bay

\section{PolSAR Unsupervised Classification of Oil Slick Thickness}

In general, according to the specific conditions of the experiment, the number of cluster categories of fuzzy C-means clustering algorithm can be given [4]. In this paper, the improved algorithm improves the selection of the initial cluster center based on the given number of categories. In the preprocessing stage of the algorithm, the point density of the sample is calculated, and some points whose point density is greater are selected as the initial cluster center to conduct the clustering algorithm. Usually, if there are denser points around a sample point, the impact of the sample point on the clustering will be greater [5]. Taking into account the correlation between samples, Mahalanobis distance is used to calculate the point density.

\section{Results and Discussion}

Effect verification of improved oil slick thickness estimation based on multi-feature. The improved $\mathrm{C}$-means fuzzy clustering algorithm is used in the paper. Based on this set of features subset (, , and ), the analysis of the quasi-synchronous dual polarization SAR and multi-spectral MODIS oil spill data is conducted; the confusion matrix is shown in Table 1. Kappa coefficient of the classification is 0.8465 based on this set of subset features. Having been mentioned in the third chapter, the feature combination is suitable for the estimation of oil slick thickness. The complementary between polarimetric features does exist.

Classification test of oil slick thicness. Based on the selected features subset, two areas of $200 * 200$ sub-images and an oil spill area of 100*100 sub-image are respectively intercepted in the Mexico Bay data (No.1). And in the second scene of data, two areas of $200 * 200$ sub-images and an oil spill area of 100*100 sub-image are also respectively intercepted. The specific classification results are shown in Fig. 5 and Fig. 6. The laws obtained by the classification results of oil slick thickness in Fig. 5 and Fig. 6 are as follows:

- In the Mexico Bay data (No.1), although the absolute thickness is unknown, it can be seen that the overall oil slick is thick (as shown in Fig. 5). The acquisition time of the Mexico Bay data (No.1) is at the early stage of the oil spill accident, while the acquisition time of the Mexico Bay data (No.2) is more than 1 year from the accident occurred, so the oil slick is thin.

- Although there are great differences in meteorological and ocean current conditions when acquiring 2 scenes of oil spill data in the Mexico Bay, the change of oil slick thickness can be reflected steadily by the feature fusion.

Summary. The classifier of multi-feature fusion strategy is used to estimate oil slick thickness. The combination of these characteristics is applied to oil spill POLSAR data of the Mexico Bay (No.1) and 
oil spill POLSAR data of the Mexico Bay (No.2). The effectiveness of the method in the paper is verified by classification experiments of oil slick thickness in the part of the sampling areas.

\section{Conclusions and Outlooks}

In this paper, PolSAR data is used to estimate the oil slick thickness. And the main work and research results include the following aspects:

- The potential relationship between partial polarization features and oil slick thickness is found by extracting and analyzing PolSAR features.

- The classifier of multi-feature fusion strategy is used to estimate oil slick thickness. Compared with the oil slick thickness of single feature estimation, the classification accuracy of multi-feature fusion is significantly improved.

Affecting by external factors, the oil slick thickness changes quickly, so it's difficult to verify it [6]. The improved fuzzy C-means clustering algorithm is an algorithm of unsupervised classification. It's an attempt to estimate oil slick thickness by POLSAR data in this paper. The potential association between polarization characteristics and oil slick thickness is found, but more discussion and research are needed about the interpretation of the imaging mechanism of PolSAR and the physical mechanism of spilled oil slick thickness.

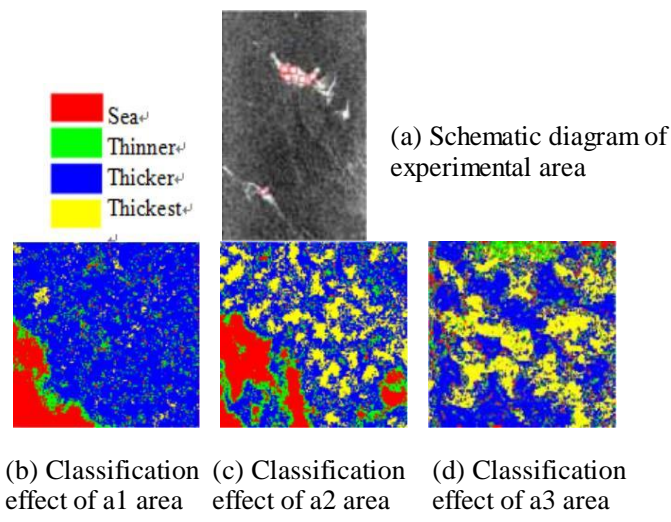

Figure 5. The classification result of the first scene data

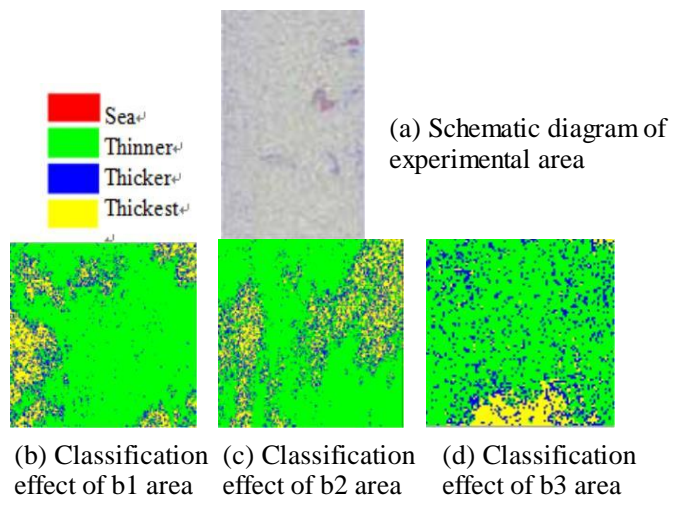

Figure 6. The classification result of the second scene data

\section{Acknowledgements}

The authors would like to thank the National Natural Science Foundation of China (Grant 61471079) and State Oceanic Administration of China for ocean nonprofit industry research special funds (No.2013418025) for providing truth data.

\section{References}

[1] Peng Liu, Study on detection and recognition method of SAR sea surface oil spill, Ph.D., Chinese Ocean University, China, 2012:4-6.

[2] P. Pavlakis, On the monitoring of illicit vessel discharges using spaceborne SAR remote sensing; a reconnaissance study in the Mediterranean sea, Annals of Telecom, vol. 56, no. 11, 2001:700-718.

[3] Reza Shirvany, Marie Chabert, Jean-Yves Tourneret, Ship and oil-spill detection using the degree of polarization in linear and hybrid/compact dual-pol SAR, IEEE, 2012:885-891.

[4] B. Fiscella, A. Giancaspro, F. Nirchio, P. Pavese, P. Trivero, Oil spill detection using marine SAR images, International Journal of Remote Sensing, 2000, 21(18): 3561-3566.

[5] Stine Skrunes,Camilla Brekke and Torbjørn Eltoft, Oil Spill Characterization with Multi-Polarization C- and X-Band SAR, IEEE, 2012:5117-5119.

[6] Deababrata Samanta and Goutam Sanyal, Segmentation Technique of SAR Imagery based on Fuzzy c-means clustering, IEEE, 2012:610-611. 